\title{
Evaluating Impacts of Time-varying Productivity in Stock-recruit Relationships on Biological Benchmarks
}

\author{
Carrie A. Holt ${ }^{1}$ and Catherine G.J. Michielsens ${ }^{2}$ \\ ${ }^{\text {I}}$ Pacific Biological Station, Fisheries and Oceans Canada, 3190 Hammond Bay Rd., Nanaimo, B.C., V9T 6N7, \\ Canada \\ ${ }^{2}$ Pacific Salmon Commission, 600-1155 Robson Street, Vancouver, B.C., Canada
}

Keywords: time-varying productivity, Ricker model, benchmarks, reference points, simulation, Kalman Filter

Time-varying models that account for changes in productivity are increasingly being applied to assess fish stocks, including Pacific salmon (Peterman et al. 2000; Dorner et al. 2008; Peterman and Dorner, 2012), but their reliability under different harvest and productivity scenarios has not been thoroughly evaluated. In particular, the Kalman filter has been used as an analytical tool to identify time-varying productivity in Pacific salmon stocks assessed with a Ricker stock-recruit relationship (Peterman et al. 2000). While variability in the underlying spawner and recruitment data, e.g., due to variability in a stock's exploitation history, may impact the relative performance of models, this has not been considered in model evaluations. In addition, the implications of time-varying productivity on the derivation of biological reference points or benchmarks have not been quantified for Pacific salmon. Here, we evaluated stock-recruitment models with and without time-varying productivity parameters in a closed-loop simulation model of sockeye salmon (Oncorhynchus nerka). This modelling approach allowed us to evaluate biases and precision of parameter estimates under various hypotheses about trajectories in productivity and exploitation rates, and their implications on biological benchmarks.

We addressed this overarching goal with the following objectives.

(1) Evaluate the bias and precision of parameter estimates from a standard stock-recruit model against one that includes time-varying productivity, using simulated stock-recruitment data based on different scenarios of temporal changes in productivity and exploitation rates.

(2) Evaluate the bias and precision of a biological benchmark, spawner abundances at maximum sustainable yield, $S_{\mathrm{MSY}}$, derived from the estimated parameters of standard and time-varying models.

(3) Provide suggestions on best practices when considering time-varying productivity in stock assessments and when deriving benchmarks.

Methods

The standard Ricker model assumes productivity remains unchanged over time and is formulated:

$$
\text { (1) } \log _{e}\left(\frac{R_{t}}{S_{t}}\right)=\alpha-\beta S_{t}+v_{t} \text {, }
$$

where $S_{\mathrm{t}}$ is the total number of spawners in the brood year $t, R_{\mathrm{t}}$ is the number of adult recruits produced by those spawners, $\alpha$ is the productivity at low spawner abundance in the absence of density dependence, $\beta$ is the rate at which recruitment is reduced by density-dependence and the inverse of the abundance of spawners at maximum recruitment or capacity, and $v_{t}$ are random normal deviations with variance $\sigma_{v}{ }^{2}, v_{t} \sim N\left(0, \sigma_{v}{ }^{2}\right)$.

We incorporated time-varying productivity into the Ricker model by allowing the productivity parameter, $\alpha$, to vary over time $t$ according to a random walk:

$$
\begin{aligned}
& \text { (2) } \log _{e}\left(\frac{R_{t}}{S_{t}}\right)=\alpha_{t}-\beta S_{t}+v_{t}, \\
& \text { (3) } \alpha_{t}=\alpha_{t-1}+\omega_{t},
\end{aligned}
$$

where $\omega_{t}$ are random normal process errors with variance $\sigma_{\omega}{ }^{2}, \omega \sim N\left(0, \sigma_{\omega}{ }^{2}\right)$. Parameters of the time-varying Ricker model (Eqns. 2 and 3) were estimated using a recursive Kalman Filter algorithm with maximum likelihood estimation (Harvey 1989; Peterman et al. 2000, Britten et al. 2016).

The Monte Carlo simulation framework used to evaluate standard and time-varying Ricker models included spawner and recruitment dynamics each year followed by the impacts of harvest on spawner abundances in the following year. We evaluated performance of standard and time-varying stock-recruitment models for tracking true underlying productivity, under three productivity scenarios (constant, declining and increasing) and three exploitation rate scenarios (low and high constant exploitation and a stepwise decline in exploitation). Our 
simulation model was a simplified version of models previously developed to evaluate benchmarks for southern British Columbia chum salmon (Holt and Folkes 2015; Holt et al. 2018), which captures the general population and management dynamics for Pacific salmon stocks.

\section{Results and Discussion}

Results indicated that time-varying models tended to perform as well as or outperform standard stockrecruitment models that did not account for time-varying productivity (e.g., Fig. 1, under scenario of underlying declines in productivity). Ignoring trends in productivity within the model led to significant over-estimates of the benchmark, $S_{\mathrm{MSY}}$ when exploitation rates were constant and high (Fig. 1). However, $S_{\mathrm{MSY}}$ was slightly underestimated by the standard model when both exploitation rates and productivity declined over time, as has been observed for Fraser River sockeye salmon. In contrast, time-varying models tended to be unbiased on average. Although models that incorporate time-varying productivity will provide biological benchmarks that are less biased relative to true underlying benchmarks, some biological benchmarks trend downwards (i.e., become less precautionary) when productivity declines as few spawners are needed to sustainably maximize yield.

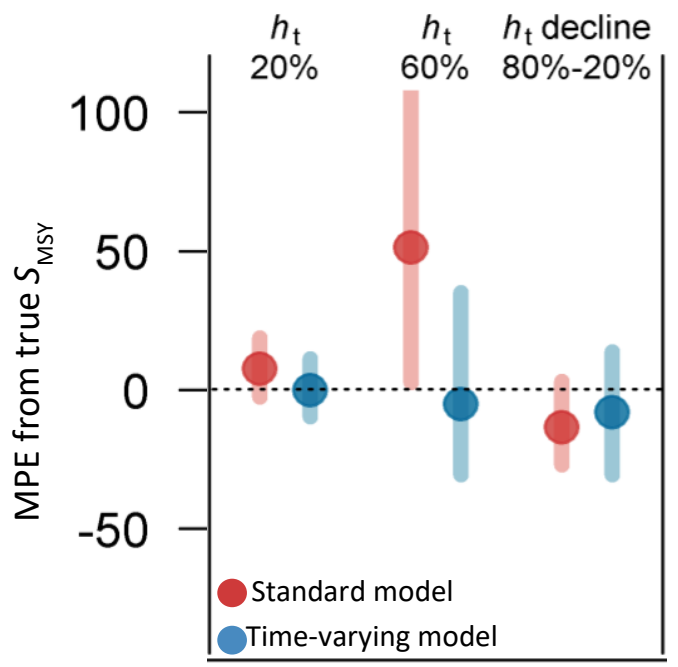

Fig. 1. Mean percent error in estimate of spawner at maximum sustainable yield, $S_{\mathrm{MSY}}$, for the standard Ricker model (red) and timevarying Ricker model (blue). Bars represent $95 \%$ confidence intervals. Results from 3 exploitation rate scenarios are shown: constant low exploitation rate $\left(h_{t} 20 \%\right)$, constant high exploitation rate $\left(h_{t} 60 \%\right)$, and a step decline in exploitation rate halfway through the time-series $\left(h_{\mathrm{t}}\right.$ decline $80 \%-20 \%$ ). The upper $97.5 \%$ upper CL for MPE of the standard model under constant high exploitation rate is above the upper limit of the plot at $182 \%$.

We suggest several best practices when considering revisions to benchmarks or biological reference points due to persistent shifts in productivity to ensure decision making is sound and transparent (adapted from Duplisea and Cardigan (2012)). These recommendations include documenting evidence of changes in exploitation of the stock, documenting evidence for the changes in productivity, calculating stock-recruitment parameter estimates and benchmarks with and without time-varying productivity and comparing values, and importantly, supporting decisions for benchmarks or reference points with simulation models that include management procedures with the assessment and application of those reference points (e.g., in harvest control rules) and uncertainty in future trends in productivity (as shown for Pacific salmon by Collie et al. (2012)).

\section{REFERENCES}

Britten, G.L., M. Dowd, and B. Worm. 2016. Changing recruitment capacity in global fish stocks. Proc. Natl. Acad. Sci. 113: 134-139.

Collie, J.S., R.M. Peterman, and B.M. Zuehlke. 2012. A fisheries risk-assessment framework to evaluate trade-offs among management options in the presence of time-varying productivity. Can. J. Fish. Aquat. Sci. 69: 209223.

Dorner, B., R.M. Peterman, and S.L. Haeseker. 2008. Historical trends in productivity of 120 Pacific pink, chum and sockeye salmon stocks reconstructed by using a Kalman filter. Can. J. Fish. Aquat. Sci. 65: 1842-1866.

Duplisea, D., and N. Cadigan. 2012. Proceedings of the national workshop for technical expertise in stock assessment (TESA): maximum sustainable yield (MSY) reference points and the precautionary approach when productivity varies. Can. Sci. Adv. Sec. Proc. Ser. 2012/055.

Harvey, A.C. 1989. Forecasting, structural time series models, and the Kalman filter. Cambridge University Press, Cambridge, U.K. 572 pp. 
Holt, C.A., B. Davis, D. Dobson, L. Godbout, W. Luedke, J. Tadey, and P. Van Will. 2018. Evaluating Benchmarks of Biological Status for Data-limited Conservation Units of Pacific Salmon, Focusing on Chum Salmon in Southern BC. DFO Can. Sci. Adv. Sec. Res. Doc. 2018/011. 77 pp.

Holt C.A., and M.J.P. Folkes. 2015. Cautions on using percentile-based benchmarks of status for data-limited populations of Pacific salmon under persistent trends in productivity and uncertain outcomes from harvest management. Fish. Res. 171: 188-200.

Peterman R.M., B.J. Pyper, and J.A. Grout. 2000. Comparison of parameter estimation methods for detecting climate-induced changes in productivity of Pacific salmon (Oncorhynchus spp.). Can. J. Fish. Aquat. Sci. 57: 181-191.

Peterman R.M., and B. Dorner. 2012. A widespread decrease in productivity of sockeye salmon (Oncorhynchus nerka) populations in western North America. Can. J. Fish. Aquat. Sci. 69: 1255-1260. 